\title{
CEREBELLOPONTINE ANGLE LESIONS - CT AND MR IMAGING EVALUATION
}

\author{
Tralukya Nandan Sonowal', Sushant Agarwal2 ${ }^{2}$ Hrishikesh Choudhury33, Nency Brahma 4
}

${ }^{1}$ Assistant Professor, Department of Radiology, Gauhati Medical College \& Hospital.

${ }^{2}$ Registrar, Department of Radiology, Gauhati Medical College \& Hospital.

${ }_{3}^{3}$ Assistant Professor, Department of Radiology, Gauhati Medical College \& Hospital.

4Junior Resident, Department of ENT, Gauhati Medical College \& Hospital.

\section{ABSTRACT}

\section{BACKGROUND}

CPA lesions are clinically nonspecific and the presenting symptoms are not related to the nature of the lesion itself, but to the nerves or cerebral structure involved with the lesions. Therefore, preoperative diagnosis of a CPA region tumoural lesion is based on imaging.

Aim of the study was to determine various CT and MR imaging features of different CPA lesions and provide accurate possible preoperative diagnosis. To propose a concise algorithm to facilitate diagnosis and to correlate histopathologically post-operatively.

\section{MATERIALS AND METHODS}

Retrospective observational study was carried out during a period from April, 2012 to March, 2015 on 62 selected patients referred from various departments with clinical suspicion, and after CT \& MRI both revealed cerebellopontine angle lesions. Lesions were retrospectively analysed according to its relative CT attenuation, MR signal intensity with morphologic appearance on T1- and T2-weighted images and contrast enhancement patterns along with diffusion and spectroscopic features.

\section{RESULTS}

Of the total 62 cases, 40 patients had undergone operative treatment and rest of 22 cases were treated conservatively and radiotherapy. Histopathological findings are correlated in 40 patients. Majority $43.5 \%$ patients were in age between $41-50$ yrs. Females predominated with $56.4 \%$ cases. Most common CPA lesion encountered was acoustic schwannoma (67.7\% cases) followed by meningioma, arachnoid cyst and epidermoid. Most common presenting symptom was hearing loss (77.4\%) followed by tinnitus (59.6\%). Most CPA masses were hypodense on unenhanced scans (64.5\%). Majority of acoustic schwannoma showed expansion of IAC (85.7\%). Hydrocephalus and calcification seen mainly in acoustic schwannoma among all CPA masses. On contrast enhanced CT and MRI, majority CPA showed heterogenous enhancement. Dural tail sign seen in 5 out of 6 cases of meningioma. On MRS of CPA with large extracanalicular component, acoustic schwannoma showed MI peak (3.55 ppm) and meningioma showed alanine peak (1.47 ppm).

\section{CONCLUSION}

Both CT and MRI can depict the characteristic imaging features, allow a systematic approach to preoperative diagnosis in majority of cases. Advanced MR imaging techniques such as diffusion weighted imaging as well as spectroscopy help to differentiate variety of lesions.

\section{KEYWORDS}

Acoustic Schwannoma, Magnetic Resonance Imaging, Cerebellopontine Angle.

HOW TO CITE THIS ARTICLE: Sonowal TN, Agarwal S, Choudhury H, et al. Cerebellopontine angle lesions - CT and MR imaging evaluation. J. Evolution Med. Dent. Sci. 2016;5(91):6750-6756, DOI: 10.14260/jemds/2016/1525

\section{BACKGROUND}

Before the advances in neuroradiologic imaging and surgical techniques, approaches to cerebellopontine angle lesions were difficult to diagnose leading to incomplete excision. The mortality rate for operative intervention in the posterior fossa approached up to 50\%. Surgical procedures or therapeutic plan of each tumorous condition may be quite different depending on the preoperative impression.[1] In present day, with better imaging modalities and surgical advancement, the lesions are almost uniformly treatable with acceptable morbidity and very low rates of mortality.

Financial or Other, Competing Interest: None.

Submission 07-10-2016, Peer Review 30-10-2016,

Acceptance 07-11-2016, Published 12-11-2016.

Corresponding Author:

Dr. Sushant Agarwal,

Registrar,

Department of Radiology,

Gauhati Medical College \& Hospital,

Guwahati-781032, Assam.

E-mail: sushantgmc@rediffmail.com

DOI: $10.14260 /$ jemds/2016/1525
The cerebellopontine (CPA) cistern is a cerebrospinal fluid (CSF) filled subarachnoid space that contains cranial nerves and vessels. The CPA is bounded by the pons, anterior aspect of the cerebellum and the petrous temporal bone covered by dura mater. It is centred by the internal auditory canal (IAC) and extends caudally from the cranial nerve $V$ to the IX-X-XI cranial nerve complex. CPA lesions are clinically nonspecific and the presenting symptoms are not related to the nature of the lesion itself, but to the nerves or cerebral structure involved with the lesions. Therefore, preoperative diagnosis of a CPA region tumour is based on imaging. Diagnosis may be difficult because of the wide variety of cell types and origins of such tumours. Because vestibular schwannoma represents for $70-80 \%$ of all CPA lesions, meningiomas $10-15 \%$ and epidermoid cysts $5 \%$, the few remaining lesions, which account for less than $1 \%$ each, are derived from a wide spectrum of lesions that are challenging to diagnose.[2,3] In addition to computed tomography and conventional magnetic resonance (MR) imaging characteristics of the different CPA lesions (Including anatomic site of origin, shape, density, signal characteristics, and behaviour pattern after contrast media injection), data 
from MR advanced techniques such as diffusion weighted imaging (DWI) and MR spectroscopy, when available, gives new crucial data allowing accurate preoperative diagnosis.

\section{Aims and Objectives}

In this study, we are

1. To determine various CT and MR imaging features of different CPA lesions and provide accurate possible preoperative diagnosis.

2. To propose a concise algorithm to facilitate diagnosis.

3. To correlate histopathologically post-operatively.

\section{MATERIALS AND METHODS}

The present study was carried out in the Department of Radiology, Gauhati Medical College and Hospital.

The retrospective observational study was carried out during a period from April, 2012 to March, 2015.

The resultant study population consisted of 62 patients referred from various departments. The cases were selected on the basis of after diagnostic tests revealed cerebellopontine angle lesions.

All the patients were clinically assessed. In all the cases, thorough history was taken including presenting complaints, duration and progression of symptoms, personal history (Including occupation, etc.), medical history and treatment history if any.

All the patients were studied, recorded according to the proforma.

Patients included in the study had undergone both CT scans \& MRI.

CT scans were obtained using Somatom spirit dual slice and 16 slice Philips MX16 CT machines. The patients underwent both unenhanced and contrast-enhanced CT. The scanning was done in the sequence axial sections with $5 \mathrm{~mm}$ slice thickness and $10 \mathrm{~mm}$ table feed. High resolution computed tomographic images were also studied in axial section of mastoid bone centring at the internal auditory canal.

MRI system using Siemens Tim Avanto 1.5 Tesla machine images were obtained with a head coil, and a adapted protocol for the cerebellopontine angle evaluation (T2 3D FSE, FLAIR, 3D FSPGR with and without Gd-DTPA, 3D ToF, diffusion, T1 without and with fat saturation, MR spectroscopy).

Lesions were retrospectively analysed according to its relative CT attenuation, MR signal intensity with morphologic appearance on T1- and T2-weighted images and contrast enhancement patterns along with diffusion and spectroscopic features.

\section{RESULTS}

The study was carried out in the Department of Radiodiagnosis, Gauhati Medical College and Hospital for a period of 3 years from April, 2012 to March, 2015.

Patients referred to the department with clinical suspicion of cerebellopontine angle cistern lesion were studied. The patients were evaluated with CT and MRI without and with intravenous contrast media. Imaging features of total number of 62 cases were evaluated. Of the total cases, 40 patients were subjected to operative treatment and rest of 22 cases were treated conservatively (Including radiotherapy). Histopathological findings were correlated in 40 patients.

\section{Inclusion Criteria}

$\mathrm{CP}$ angle lesions with features of neoplastic, vascular masses and secondary involvement by neoplastic processes were included in the study.

\section{Exclusion Criteria}

$\mathrm{CP}$ angle lesions occurring from infectious processes were excluded from analysis.

The results observed in the present study were Age incidence - In this study group of 62 patients, the various age groups were as follows:

\begin{tabular}{|c|c|c|}
\hline Age in Years & No. of Patients & Percentage (\%) \\
\hline $0-10$ & 1 & 1.6 \\
\hline $11-20$ & 4 & 6.4 \\
\hline $21-30$ & 8 & 12.9 \\
\hline $31-40$ & 11 & 17.5 \\
\hline $41-50$ & 27 & 43.5 \\
\hline $51-60$ & 6 & 9.6 \\
\hline $61-70$ & 3 & 4.8 \\
\hline $71-80$ & 1 & 1.6 \\
\hline $81-90$ & 1 & 1.6 \\
\hline \multicolumn{3}{|c|}{ Table 1a. Showing Distribution of } \\
CPA Cases According to Age \\
\hline
\end{tabular}

So the maximum numbers of cases were seen in the $5^{\text {th }}$ decade numbering 27 cases (43.5\%). Out of total 62 cases, acoustic schwannoma accounted for the highest number of cases -42 cases $(67.7 \%)$.

\section{Sex Distribution}

In our present series, 62 patients with cerebellopontine angle lesions were evaluated, out of which 27 patients (43.5\%) were male and 35 patients $(56.4 \%)$ were females. Thus, we found female predominance with male: female ratio of 1:1.3.

\begin{tabular}{|c|c|c|}
\hline Sex & No. of Cases & Percentage \\
\hline Male & 27 & 43.5 \\
\hline Female & 35 & 56.4 \\
\hline \multicolumn{3}{|c|}{ Sex Incidence of CPA Lesion } \\
\hline
\end{tabular}

Age incidence of Acoustic Schwannoma

\begin{tabular}{|c|c|}
\hline Age in Years & Acoustic Schwannoma \\
\hline $0-20$ & 2 \\
\hline $21-40$ & 14 \\
\hline $41-60$ & 25 \\
\hline $61-80$ & 1 \\
\hline $\begin{array}{c}\text { Table 1b. Showing Distribution of Acoustic } \\
\text { Schwannoma cases }\end{array}$ \\
\hline
\end{tabular}

So out of 42 diagnosed acoustic schwannoma cases, the maximum number of cases (25) were seen in the $5^{\text {th }}$ to $6^{\text {th }}$ decade followed by $3^{\text {rd }}$ to $4^{\text {th }}$ decade numbering 14 cases.

Sex incidence of Acoustic Schwannoma

\begin{tabular}{|c|c|c|}
\hline Sex & No. of Cases & Percentage \\
\hline Male & 18 & 42.9 \\
\hline Female & 24 & 57.1 \\
\hline \multicolumn{2}{|c|}{ Table 2. Showing Distribution of Acoustic } \\
Schwannoma cases According to Sex \\
\hline
\end{tabular}


Clinical Presentations

\begin{tabular}{|c|c|}
\hline Symptoms & No. of Cases \\
\hline Hearing loss & 48 \\
\hline Tinnitus & 37 \\
\hline Vertigo & 32 \\
\hline Headache & 16 \\
\hline Papilloedema & 3 \\
\hline Vomiting & 5 \\
\hline Trigeminal neuralgia & 1 \\
\hline \multicolumn{2}{|c|}{$\begin{array}{c}\text { Table 3. Showing Distribution of CPA cases } \\
\text { According to Clinical Presentations }\end{array}$} \\
\hline
\end{tabular}

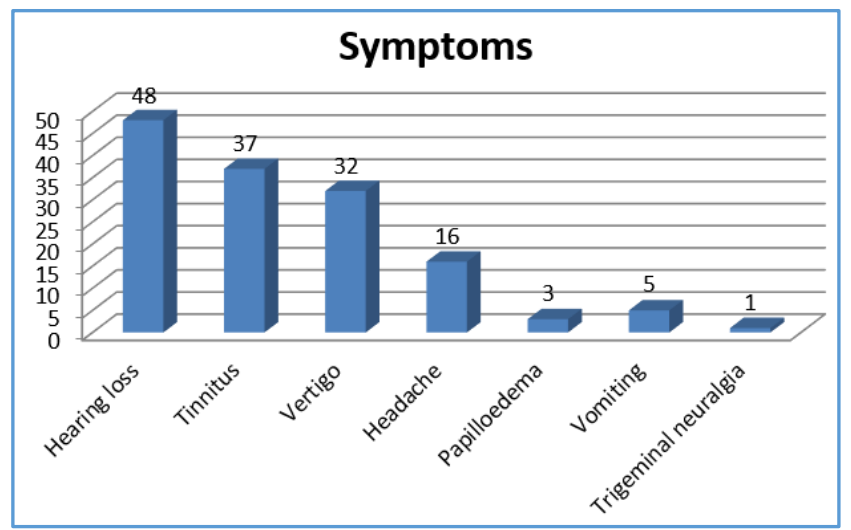

Figure 1. Bar Diagram Showing Distribution of CPA cases According to Clinical Presentations.

Thus, hearing loss, tinnitus, vertigo and headache are the most common presenting symptoms of cerebellopontine angle lesions respectively.

CT and MRI Diagnosis of Cerebellopontine Angle Lesions

\begin{tabular}{|c|c|}
\hline Diagnosis & No. of Cases \\
\hline Acoustic schwannoma & 42 \\
\hline Ependymoma & 1 \\
\hline Meningioma & 6 \\
\hline Epidermoid & 4 \\
\hline Arachnoid cyst & 5 \\
\hline Lipoma & 2 \\
\hline Vertebrobasilar dolichoectasia & 2 \\
\hline \multicolumn{2}{|c|}{ Table 4. Showing Distribution of Type of } \\
CPA cases According to CT \& MRI Diagnosis \\
\hline
\end{tabular}

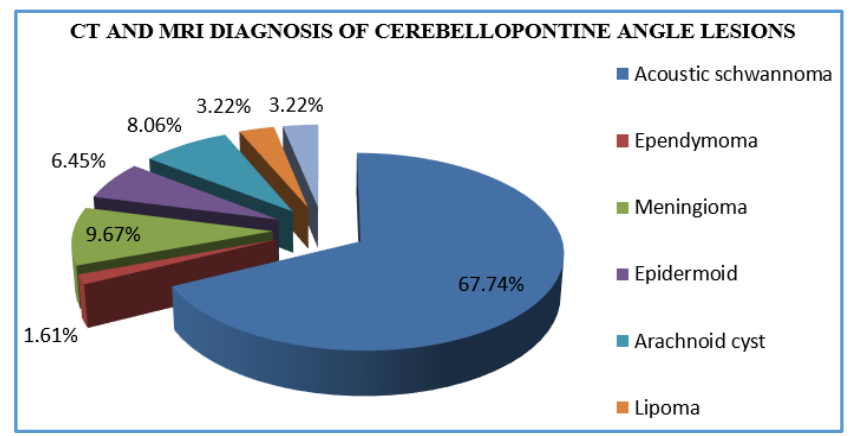

Figure 2. Pie Chart Showing Distribution of type of CPA Cases According to CT \& MRI Diagnosis

Retrospective analysis of all CPA cases showed a predominance of the schwannoma (42 cases) followed by meningioma (6 cases), arachnoid cyst ( 5 cases), epidermoid cyst ( 4 cases), vasculo-nervous conflicts ( 2 cases), lipoma of the IAC ( 2 cases) and ependymoma ( 1 case).

\section{Imaging Findings}

CT Density of the Lesions

Density of the cerebellopontine angle masses in relation to the brain parenchyma in non-enhanced CT scans.

\begin{tabular}{|c|c|c|}
\hline Density & No. & Percentage (\%) \\
\hline Hypodense & 40 & 64.5 \\
\hline Isodense & 2 & 3 \\
\hline Hyperdense & 5 & 8 \\
\hline Mixed & 15 & 35.7 \\
\hline \multicolumn{2}{|c|}{$\begin{array}{c}\text { Table 5a. Showing Distribution of CPA } \\
\text { Cases According to CT Density }\end{array}$} \\
\hline
\end{tabular}

Density of the cerebellopontine angle masses in relation to the brain parenchyma in non-enhanced CT scans done in 42 cases of acoustic schwannoma in our study.

\begin{tabular}{|c|c|c|}
\hline Density & No. of cases & Percentage (\%) \\
\hline Hypodense & 29 & 69 \\
\hline Isodense & 2 & 4.7 \\
\hline Hyperdense & 2 & 4.7 \\
\hline Mixed & 9 & 21.4 \\
\hline Table 5b. Showing Distribution of Acoustic \\
Schwannoma cases According to CT Density \\
\hline
\end{tabular}

Expansion of the Internal Auditory Canal in Cases of Acoustic Schwannoma

\begin{tabular}{|c|c|}
\hline IAC & Acoustic Schwannoma \\
\hline Expansion & $36(85.7 \%)$ \\
\hline Non expansion & $6(14.3 \%)$ \\
\hline \multicolumn{2}{|c|}{ Table 6a. Showing Distribution of Acoustic } \\
Schwannoma cases Causing Expansion of IAC \\
\hline
\end{tabular}

Presence of Extracanalicular Component in IAC in Cases of Acoustic Schwannoma

\begin{tabular}{|c|c|c|}
\hline $\begin{array}{c}\text { Size of } \\
\text { Tumour }\end{array}$ & $\begin{array}{c}\text { Intracanalicular } \\
\text { Component only }\end{array}$ & $\begin{array}{c}\text { With Extracanalicular } \\
\text { Component }\end{array}$ \\
\hline$<1.5 \mathrm{~cm}$ & 6 & 1 \\
\hline$>1.5 \mathrm{~cm}$ & 0 & $36(85.7 \%)$ \\
\hline Table 6b. Showing Distribution of Acoustic Schwannoma \\
Cases with Presence of Extracanalicular Component in IAC \\
\hline
\end{tabular}

Presence of Hydrocephalus

\begin{tabular}{|c|c|c|}
\hline $\begin{array}{c}\text { Acoustic } \\
\text { Schwannoma }\end{array}$ & Ependymoma & Meningioma \\
\hline 36 & 1 & 5 \\
\hline \multicolumn{2}{|c|}{ Table 6c. Showing Distribution of Acoustic } \\
Schwannoma Cases causing Hydrocephalus \\
\hline
\end{tabular}

\section{Presence of Calcifications}

\begin{tabular}{|c|c|}
\hline Diagnosis & Calcification \\
\hline Acoustic schwannoma & 28 \\
\hline Meningioma & 2 \\
\hline Epidermoid & 0 \\
\hline Arachnoid cyst & 0 \\
\hline Lipoma & 0 \\
\hline Vertebrobasilar dolichoectasia & 0 \\
\hline \multicolumn{2}{|c|}{$\begin{array}{l}\text { Table 7. Showing Distribution of CPA } \\
\text { Cases with Presence of Calcification }\end{array}$} \\
\hline
\end{tabular}


Post Contrast Enhancement in CT

Of the 62 CPA cases in which contrast enhanced CT scans were performed, intense homogeneous enhancement was seen in 5 lesions (8\%), 45 lesions (72.5\%) revealed heterogeneous moderate enhancement, 2 lesions(3.2\%) revealed mild enhancement and 11 lesions (17.7\%) showed no enhancement.

Post Contrast Enhancement in Contrast Enhanced T1 Weighted Images (MRI)

Of the 62 cases of the CPA angle masses, in which contrast enhanced MR study was performed, 6 lesions (9.6\%) revealed intense homogeneous enhancement, 43 lesions (69.3\%) revealed heterogeneous moderate enhancement, 2 lesions (3.2\%) revealed mild enhancement and 11 lesions (17.74\%) no enhancement.

Enhancement Pattern of Acoustic Schwannoma in CT Scan

On CECT of the 42 cases which were diagnosed as acoustic schwannoma, 4 cases $(9.5 \%)$ revealed intense enhancement, 31 cases $(73.8 \%)$ revealed moderate heterogeneous enhancement and 7 cases (16.6\%) revealed mild heterogeneous enhancement.

\section{Enhancement Pattern of Acoustic Schwannoma in MRI}

On CEMR of the 42 cases which were diagnosed as acoustic schwannoma, $32 \quad(76.3 \%)$ cases revealed moderate heterogeneous enhancement, $8(19.04 \%)$ cases revealed homogeneous enhancement and 2 (4.7\%) cases showed cystic pattern of enhancement.

Dural tail sign was recognised on MRI in 5 out of 6 cases of diagnosed meningioma.

Spectroscopic (MRS) Findings in Schwannoma and Meningioma

Lesions with large extra-canalicular component were evaluated with MRS. 35 patients with large extracanalicular component diagnosed as schwannomas revealed myoinositol peak at $3.55 \mathrm{ppm}$ in 22 cases (62.8\%). In lesions with features of meningioma, alanine peaks (1.3-1.5 ppm) were demonstrated in 3 cases (50\%).

Histopathological Findings (Correlated in 40 patients only)

\begin{tabular}{|c|c|}
\hline Acoustic Schwannoma & $25(62 \%)$ \\
\hline Meningioma & $5(12.5 \%)$ \\
\hline Epidermoid cyst & $4(10 \%)$ \\
\hline Lipoma & $2(5 \%)$ \\
\hline Arachnoid cyst & $3(7.5 \%)$ \\
\hline Ependymoma & $1(2.5 \%)$ \\
\hline \multicolumn{2}{|c|}{ Total } \\
Table 8. Showing Distribution of type of CPA cases \\
According to Histopathological Diagnosis \\
\hline
\end{tabular}
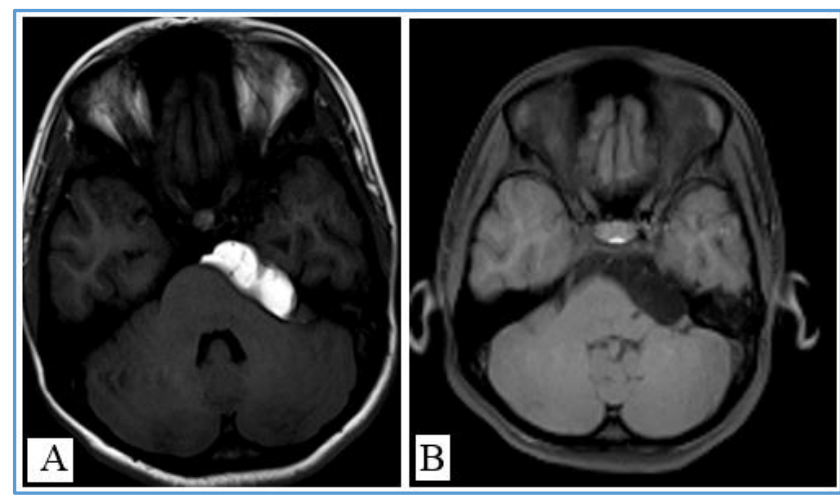

Figure 3. Axial T1 Weighted Image (A) showing Hyperintense CPA Lesion which Suppresses on Axial T1 Fat Saturated Image (B) Suggestive of Lipoma
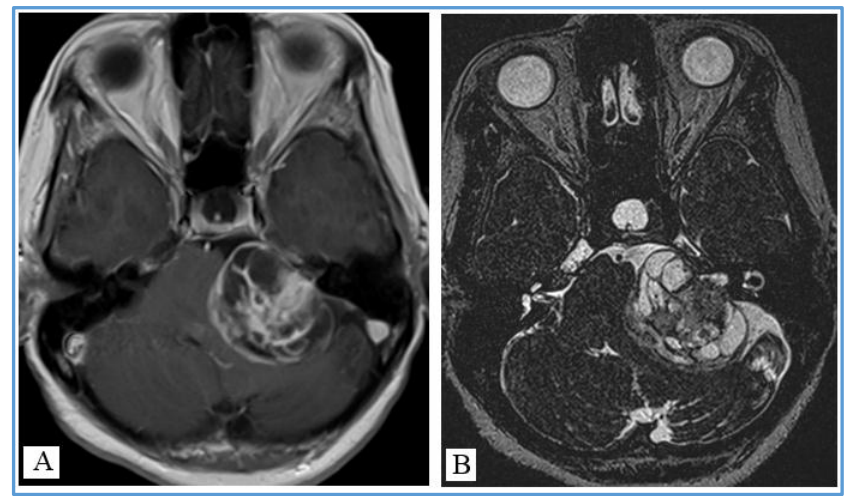

Figure 4. A) Axial T1 post Gd Enhanced Image of Acoustic Schwannoma showing Heterogeneous Enhancement and Cystic Changes. B) Axial CISS Image of Acoustic Schwannoma showing Expansion of IAC by Large Extracanalicular Component with Cystic Changes

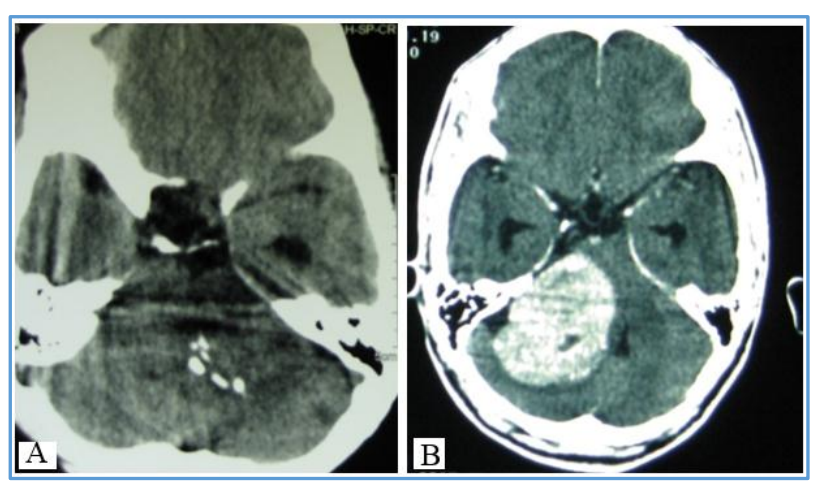

Figure 5. A) Axial NECT Scan showing Calcification in a Case of Acoustic Schwannoma. B) CECT Image showing Homogeneously Intensely Enhancing right CP Angle Meningioma 

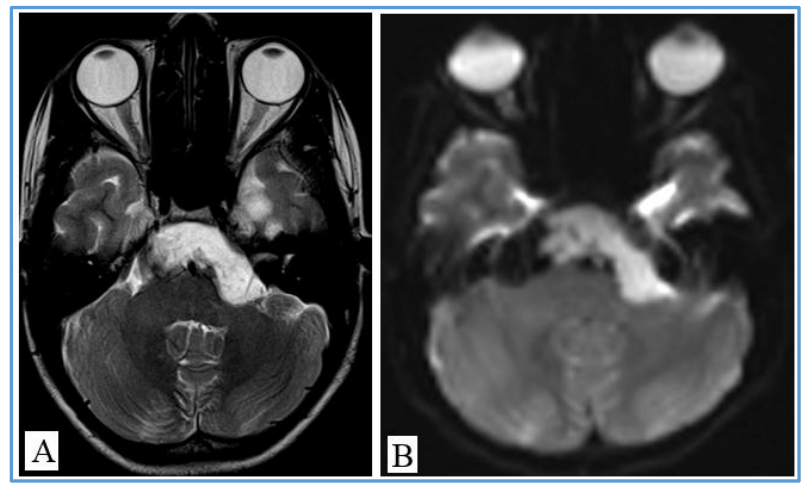

Figure 6. A) Axial T2 Weighted Image showing Insinuating Hyperintense Lesion which Follows CSF Signal on all Sequences but shows Restricted Diffusion on DWI (B) Suggestive of Epidermoid (Thus, Differentiating it from Arachnoid Cyst)
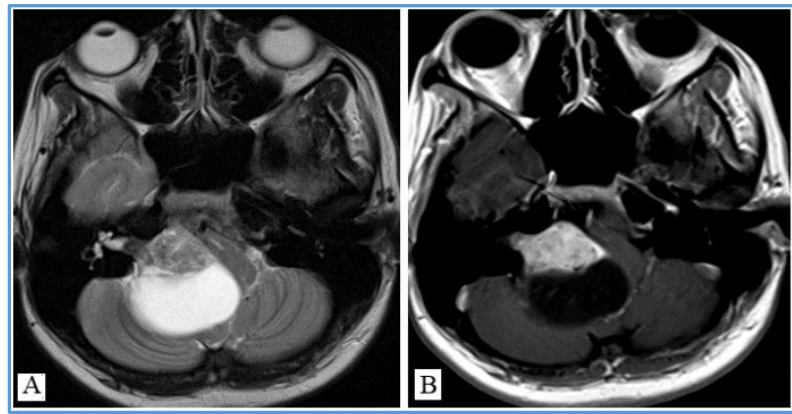

Figure 7. A) Axial T2 Weighted Image showing Acoustic Schwannoma with Large Cystic Component. B) Axial T1 Weighted Post Gadolinium Image showing Enhancing Intracanalicular Component with Expansion of IAC
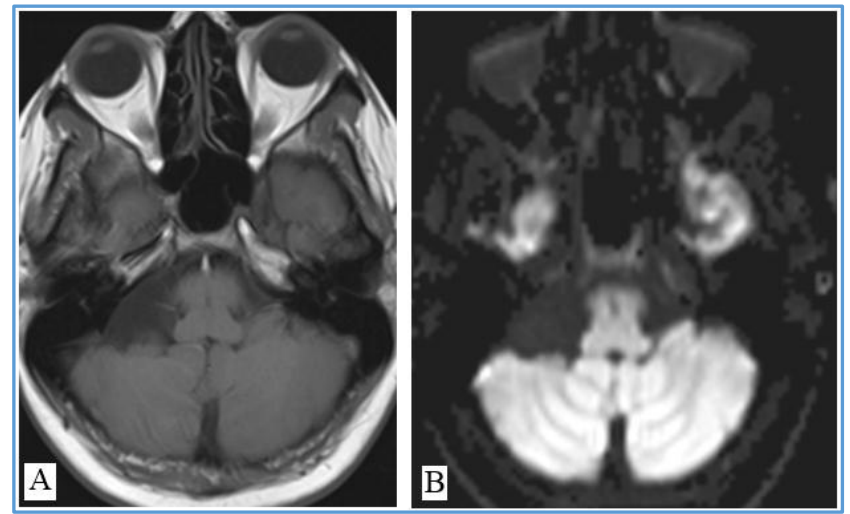

Figure 8. A) Axial T1 Weighted Image showing Hypointense Lesion in Right CP Angle with no Restricted Diffusion (B) Suggestive of Arachnoid Cyst

\section{DISCUSSION}

The lesions occurred most frequently in our series in the fifth decade consisting of $43.5 \%$ of the cases. Our study correlates well the study by Okazaki $\mathrm{H}$. et al 1989[4] who found that the maximum number of cases presented in the fourth and fifth decades.

In our study of acoustic schwannoma which constituted $67.7 \%$ of the cases, maximum no. of cases were found in the age group of $5^{\text {th }}$ to $6^{\text {th }}$ decade.

In our study, we saw a female predominance similar to study by Kasantikul $\mathrm{V}$ et al[5] where they reported male: female ratio of $1: 2$
The commonest presenting symptoms of the patients with cerebellopontine angle lesions in our study was hearing loss which accounted for $77.4 \%$ followed by tinnitus (59.6\%), vertigo (51.6\%) and headache $(25.8 \%)$. No patient was asymptomatic. Matsuka Y.et al 2000[6]reported in their study, the clinical symptoms of the $\mathrm{CP}$ angle lesions as hearing deficits (60 to $97 \%$ ), tinnitus (50 to $66 \%$ ), vestibular disturbances ( 46 to $59 \%$ ), numbness or tingling in the face (33\%), headache (19 to $29 \%$ ), dizziness (23\%), facial paresis $(17 \%)$, and trigeminal nerve disturbances (hyperesthesia, paraesthesia, and neuralgia) (12 to $45 \%$ ).

In our study of total 62 CPA lesions, 42 cases were of acoustic schwannoma constituting $67.7 \%$, followed by 6 cases of meningioma, 5 cases of arachnoid cyst, 4 cases of epidermoid, two cases of lipoma and vertebrobasilar dolichoectasia and one case of ependymoma. This correlates well with the study by Cosnard G et al 1992[7] who reported that acoustic neuromas represent $75 \%$ of all tumours of the cerebellopontine angle. Other tumours are rare and essentially consist of meningioma, detected in 10 to $14 \%$ of cases. Similarly, Swartz JD.et al 2004[8]in his study, reported that Schwannoma of the eighth cranial nerve (Acoustic neuroma) is by far the most common cerebellopontine angle lesion. Lunardi P. et al 1991 [9] also reported that acoustic schwannomas are the most common lesions, $59 \%$ of all space occupying lesions in the cerebellopontine angle and $80-90 \%$ of all tumours.

In our study on unenhanced CT scans, majority of the CPA lesions (Predominantly schwannoma) appeared hypodense compared with the attenuation of the brain. On CECT, schwannoma showed varied enhancement, with the larger tumours showing variable and heterogeneous features, including cystic changes.

Magnetic resonance imaging is a non-invasive and safe imaging modality and in current clinical practice, MRI is the first-line investigation for the identification of suspected acoustic schwannoma in appropriately selected patients.[10,11]

Three different MRI appearances of the tumoural enhancement are described in vestibular schwannomas: homogeneous (50-60\%), heterogeneous (30-40\%), and cystic (5-15\%).[12,13,14]

At present, the definitive examination is a Gd enhanced magnetic resonance scan. This can detect lesions of $2 \mathrm{~mm}$ in diameter and probably smaller.[11] In our study on unenhanced T1-weighted images, schwannomas appeared isointense to slightly hypointense relative to the brain which after gadolinium infusion, revealed moderate heterogeneous enhancement. These features are consistent with the reports by Watabe Tet al. 1989.[15]

There were signs of IAC erosion and enlargement. Erosion of the IAC indicates a schwannoma in up to $93 \%$ of cases as reported by Kendall Bet al 1977.[16] The finding of IAC expansion and erosion by acoustic schwannoma in our study was $85.7 \%$

Smirniotopoulos JG et al 1993[12] also found that schwannomas are enhancing, round masses, most commonly arising from the vestibular nerve near the porus acusticus and associated with enlargement of the internal auditory canal.

Meningiomas showed homogeneous hyperattenuation, compared with the brain on unenhanced CT scans. Meningiomas appeared isointense relative to the brain on T1- 
weighted images and showed homogeneous bright enhancement with gadolinium (On MR images) and iodine (on CT scans) with lack of IAC enhancement. These features correlates with the studies reported by Price ACet al 1989.[17]

Dural tail sign was recognized on MRI in 5 out of 6 cases of diagnosed meningioma in our study which correlated to the study by Lalwani AK et al. 1992[18]\& Goldsher D et al 1990).[19]

Proton MR spectroscopy (MRS), is limited because of the frequent lipid contamination in spectra of extra-axial lesions abutting fatty bony limits of posterior fossa. However, in large lesions of CPA it may help in distinguishing schwannomas from meningiomas by showing a prominent myo-inositol peak at 3.55 in schwannomas.[19] The combination of elevated glutamate/glutamine and the characteristic presence of alanine at $1.5 \mathrm{ppm}$ are considered very specific for meningiomas as described by Cho YD et al 2003.[19]

Epidermoids appeared as insinuating homogeneous nonenhancing masses of attenuation values near that of water on CT. On MR they appear low-to-intermediate signal intensity on T1-weighted MR images \& having high signal intensity on T2-weighted images similar to arachnoid cyst. However, these lesions showed restriction on diffusion weighted images, thus differentiating it from arachnoid cyst which follow CSF signal on all sequence. These features were consistent as reported in studies by Kendall B et al 1977, Steffey DJ et al 1988,[20] Horowitz et al 1990.[21]

Arachnoid cysts show attenuation and signal intensity parallel to those of cerebrospinal fluid, and sharp margins without calcification or contrast enhancement and without the evidence of restriction on diffusion weighted images as reported in studies by Higashi et al 1992.[22]

As reported by Smirniotopoulos et al 1993,[23],vertebrobasilar dolichoectasia have prominent flow voids on magnetic resonance images with similar finding in our study.

In our study, there were two cases of cerebellopontine angle lipoma which correlate with clinical symptoms and imaging features as reported study by Zimmermann $\mathrm{M}$ et al (1999).[24]They found that intracranial lipomas located in the cerebellopontine angle are extremely rare with slowly progressive neurological symptoms. Also, CPA lipoma on T1weighted magnetic resonance imaging revealed hyperintense lesion and non-enhancement after application of gadolinium along with fat suppression on fat saturated techniques.

\section{CONCLUSION}

An elaborate analysis of site of origin, shape, density, signal intensities and pattern of enhancement of intravenous contrast material injection allows a systematic approach to preoperative diagnosis in majority of cases. Advanced MR imaging techniques such as diffusion weighted imaging as well as spectroscopy help to differentiate variety of lesions.

Based on MRI appearances, we propose different pathologies to be described into following groups:

\section{Solid Extra-axial}

A) Enhancing masses

B) Non-enhancing lipomatous masses
Schwannoma, Meningioma. Lipoma, dermoidcyst, cholesterol granuloma.

\begin{tabular}{|l|l|}
\hline $\begin{array}{l}\text { Non-enhancing cystic } \\
\text { masses }\end{array}$ & Arachnoid cyst, Epidermoid cyst \\
\hline Intra-axial masses & Ependymoma, glioma \\
\hline Flow void masses & Vascular conflict, aneurysms, etc. \\
\hline
\end{tabular}

With the help these imaging modalities provide crucial information to deduce correct diagnosis non-invasively and proper preoperative planning.

\section{Abbreviations}

CPA: Cerebellopontine angle, CSF: Cerebrospinal fluid, IAC: Internal auditory canal, MRI: Magnetic resonance imaging, CT: Computed tomography, NECT: Non-enhanced computed tomography, CECT: Contrast enhanced computed tomography, CEMR: Contrast enhanced MRI, DWI: Diffusion weighted image, CISS: Constructive interference steady state, MRS: Magnetic resonance spectroscopy, Gd: Gadolinium contrast.

\section{REFERENCES}

1. Ojemanm RG. Surgical principles in the management of brain tumor. In: Kaye AM, Laws ER. (eds). Brain tumor. Churchill Livingstone 1995:293-304.

2. Bonneville F, Savatovsky J, Chiras J. Imaging of cerebellopontine angle lesions: an update. Part 1: enhancing extra-axial lesions. Eur Radiol 2007;17(10):2472-82.

3. Sarrazin JL. Infra tentorial tumors. J Radiol 2006;87(6Pt2):748-63.

4. Okazaki H. Fundamentals of neuropathology. 2nd edn. New York NY: Igaku-Shoin 1989.

5. Kasantikul V, Netsky MG, Glasscock ME, et al. Acoustic neurilemmoma: clinicoanatomic study of 103 patients. J Neurosurg 1980;52(1):28-35.

6. Matsuka Y, Fort ET, Merrill RL. Trigeminal neuralgia due to an acoustic neuroma in the cerebellopontine angle. J Orofac Pain 2000;14(2):147-51.

7. Cosnard G, Galidie G, Cordoliani YS, et al. Rare tumors of the cerebellopontine angle. Annales de Radiologie 1992;35(1-2):36-43.

8. Swartz JD. Lesions of the cerebellopontine angle and internal auditory canal: diagnosis and differential diagnosis. Semin Ultrasound CT MR 2004;25(4):332-52.

9. Lunardi P, Missori P, Mastronardi L, et al. Cystic acoustic schwannomas. Acta Neurochirurgica (Wien) 1991;110(3-4):120-3.

10. Bloomer CW, Ackerman A, Bhatia RG. Imaging for spine tumors and new applications. Top Magn Reson Imaging 2006;17(2):69-87.

11. Fortnum $\mathrm{H}, \mathrm{O}^{\prime} \mathrm{Neill} \mathrm{C}$, Taylor R, et al. The role of magnetic resonance imaging in the identification of suspected acoustic neuroma: a systematic review of clinical and cost effectiveness and natural history. Health Technol Assess 2009;13(18):1-154.

12. Delsanti C, Regis J. Cystic vestibular schwannomas. Neurochirurgie 2004;50(2-3 Pt 2):401-6.

13. Gomez-Brouchet A, Delisle MB, Cognard C, et al. Vestibular schwannomas: correlations between magnetic resonance imaging and histopathologic appearance. Otol Neurotol 2001;22(1):79-86.

14. Charabi S, Tos M, Thomsen J, et al. Cystic vestibular schwannoma--clinical and experimental studies. Acta Otolaryngol Suppl 2000;543:11-13. 
15. Watabe T, Azuma $\mathrm{T}$. $\mathrm{T} 1$ and $\mathrm{T} 2$ measurements of meningiomas and neuromas before and after Gd-DTPA. AJNR 1989;10:463-70.

16. Kendall B, Symon L. Investigation of patients presenting with cerebellopontine angle syndromes. Neuroradiology 1977;13(2):65-84.

17. Price AC, Runge VM, Carollo BR, et al. Contrast enhancement in the evaluation of the cerebellopontine angle. AJNR 1989;10:879.

18. Lalwani AK. Meningiomas, epidermoids, and other nonacoustic tumors of the cerebellopontine angle. Otolaryngol Clin North Am 1992;25(3):707-28.

19. Cho YD, Choi GH, Lee SP, et al. (1)H-MRS metabolic patterns for distinguishing between meningiomas and other brain tumors. Magn Reson Imaging 2003;21(6):663-72.
20. Steffey DJ, Dc Filipp GJ, Spent T, et al. MR imaging of primary epidermoid tumors. J Comput Assist Tomogr 1988;12(3):438-40.

21. Horowitz BL, Chari MH, James R, et al. MR of intracranial epidermoid tumors: correlation of in vivo imaging with in vitro 13C spectroscopy. AJNR 1990;11:299-302.

22. Higashi S, Yamashita J, Yamamoto Y, et al. Hemifacial spasm associated with a cerebellopontine angle arachnoid cyst in a young adult. Surg Neurol 1992;37(4):289-92.

23. Smirniotopoulos JG, Yue NC, Rushing EJ. Cerebellopontine angle masses: radiologic-pathologic correlation. Radiographics 1993;13(5):1131-47.

24. Zimmermann M, Kellermann S, Gerlach $\mathrm{R}$, et al. Cerebellopontine angle lipoma: case report and review of the literature. Acta Neurochir (Wien) 1999;141(12):1347-51. 